\title{
PLASTIC TREATMENT AND MICROPLASTIC PRODUCTION, AND THEIR PRESENCE IN FEED AND FOOD
}

Juan A. Conesa*

Full Professor of Chemical engineering

Director of the Research Group "Waste, Energy, Environment and Nanotechnology (WEEN)"

University of Alicante

\section{ABSTRACT}

This preprint is focused in the presence of plastics and microplastics in food. We will discuss how many we eat, and how they arrive to the food, and why. We will treat many other things, such as the waste treatment in Europe and in Spain, with updated data; how much plastic waste is generated; what are microplastics and how they are analyzed, I will tell about the experience we have at the University of Alicante (UA); how they can be removed and we will estimate how many we eat over the course of a year.

\section{WASTE TREATMENT}

Let's start by presenting data on waste production. With the data available at Eurostat [1] it is estimated that the average annual amount of waste generated, per capita, in Europe is $486 \mathrm{~kg}$. Right in the middle is Spain, which we will later compare with plastic waste. The big producers, per capita, would be Denmark, Cyprus, Germany, Luxembourg, Montenegro and Austria. The least generating (per capita) are Romania, Poland and the Czech Republic.

In general, in Europe, in treatment centers, a pattern is followed that would be given by the scheme in Figure 1. Of course, we are talking about waste that is treated; Another thing is the existence of illegal landfills that, as their name or name indicates, are illegal and therefore uncontrollable. In some press headline I have come to see a figure of 1582 (!) Illegal dumps. I wonder how the journalist has counted them since if they are illegal, they are not counted anywhere.

At first, these treatment plants (Figure 1) the wastes are passed through a size selector which selects what will be called organic fraction. This fraction is taken to biomethanizers and/or composting. Then the glass is separated and as products there would be gas (which can be used to generate electricity), and compost ("stabilized solid" more properly said) that serves for agricultural amendment.

Then, the non-organic fraction follows a selection process, manual and with the help of optical readers, so that various plastics and metals are separated. Finally, with what is called the "rest fraction", you must choose either incineration or its discharge. Then we'll see what percentage is held each one of these purposes.

*email: ja.conesa at ua.es 


\section{Municipal solid waste treatment}

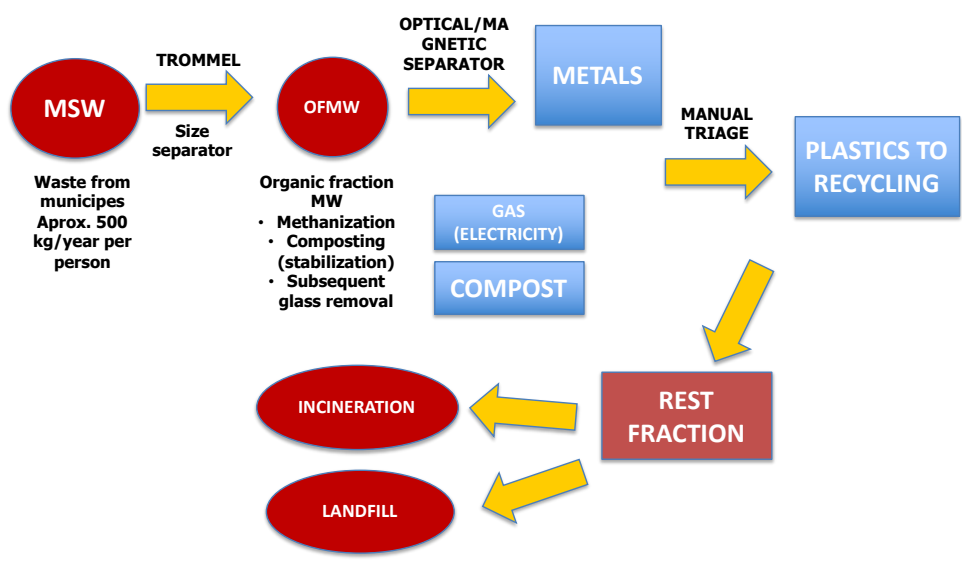

Figure 1.

What does the European regulation tell us? There is a 2008 regulation [2], still in force, which says that the first thing we have to do is reduce the production of waste, which is why it is larger in the pyramid (Figure 2). Then, if the waste is produced, it must be reused (giving it the same use as the original). If it cannot be reused, it must be recycled, which involves more complex operations that a new utility to the residue. And finally, if it cannot be recycled, it must be valued, even energetically, which is what takes place in incinerators. And finally, the fraction that cannot be valued must be buried in landfills.

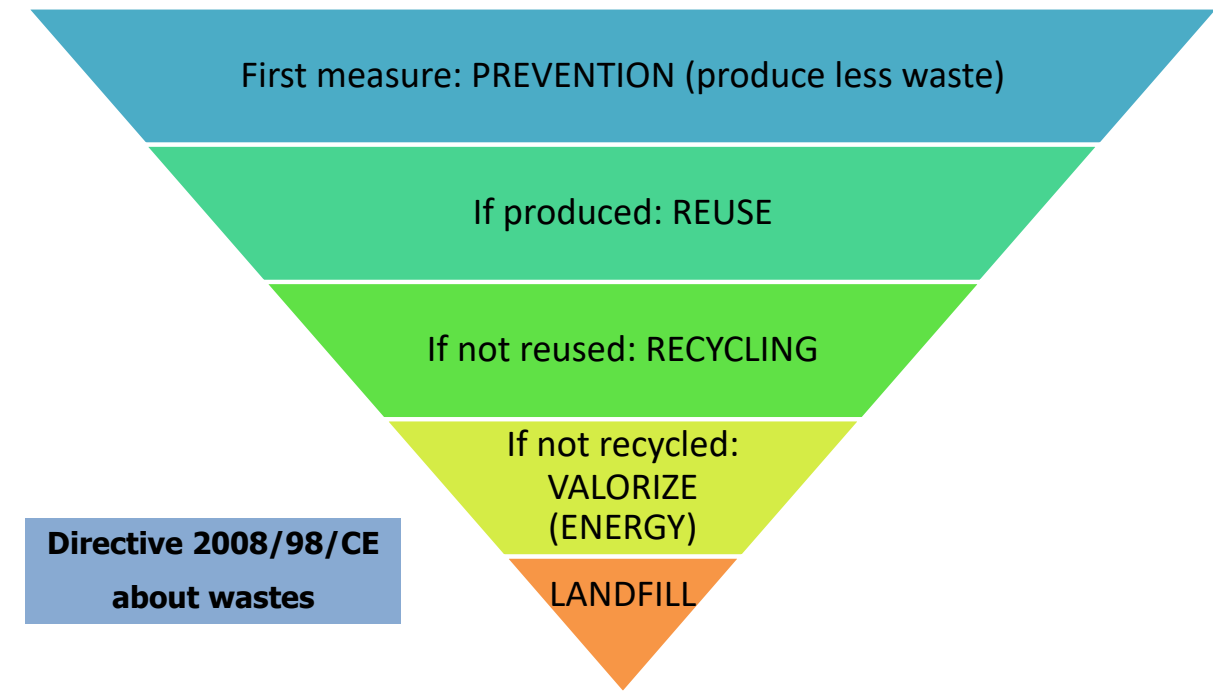

Figure 2.

What happens in Spain? In Spain and also in other European countries, the pyramid is, somehow, inverted, so that almost the first thing they do with a waste is to take it to the landfill (we will see the figures now), there is little recovery, some recycling, quite little reuse and very little prevention in the generation of waste. An example is the indiscriminate use of plastics to package even individualized fruits.

How has waste treatment evolved in Europe? In general, in Europe as a whole, it can be verified [1] that there has been, in the period 1995-2017, a decrease in landfill, and an increase in composting 
(and methanization), incineration and recycling. Looking at it by country we have some with more than $58 \%$ recycling, and Spain, for example, is in the less than $28 \%$ area.

The EU has set targets, which are the reuse and recycling of at least $55 \%$ of household waste, by 2025 , and reduce landfill to less than $10 \%$ in 2035 . The current situation in Spain is $30 \%$ recycling including composting and methanization (that is, it is not comparable to the $55 \%$ target), since there is talk of waste reuse processes. The landfill target is less than $10 \%$, with Spain currently at $58 \%$.

Figure 3 shows the methods of waste treatment and the generation of household waste per capita in different EU countries. If we try to make correlation between countries most waste-producers and incineration, we see that in many cases is not consistent. This is an argument that the environmental sector tends to use, claiming that the countries that incinerate the most are less careful to produce little waste, but we see that this is not true. There are countries that do, but many others that do not. We see that there are countries with a high rate of waste generation (red line) that have high discharge percentages, and others that have high incineration rates. There is no correlation.

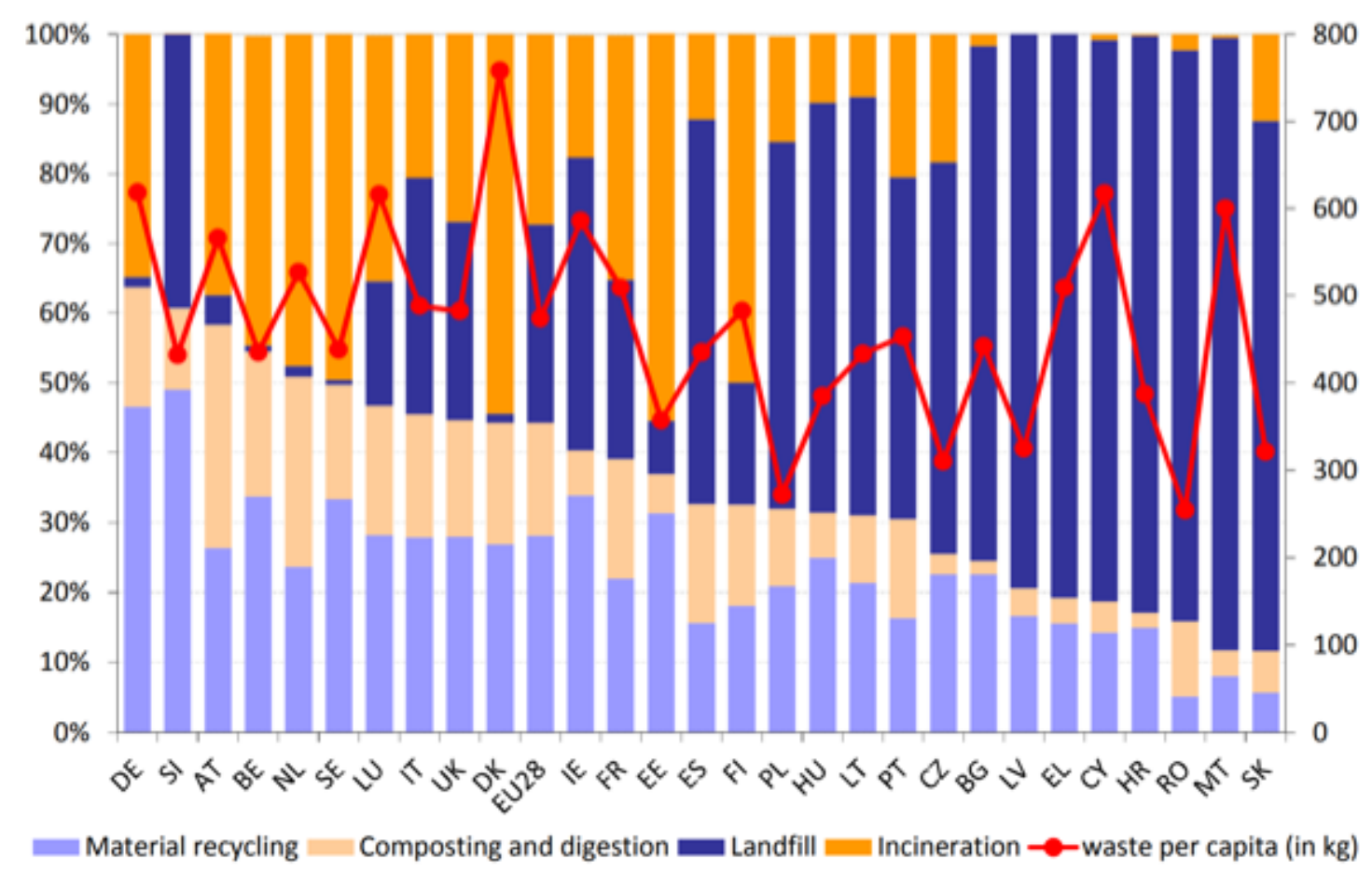

Figure 3.

Where there is a correlation is between the per capita income of a country, and the amount of waste it incinerates, with some exceptions (Figure 4). We see that the countries with the highest per capita income that tend to optimal waste management by introducing incineration. 


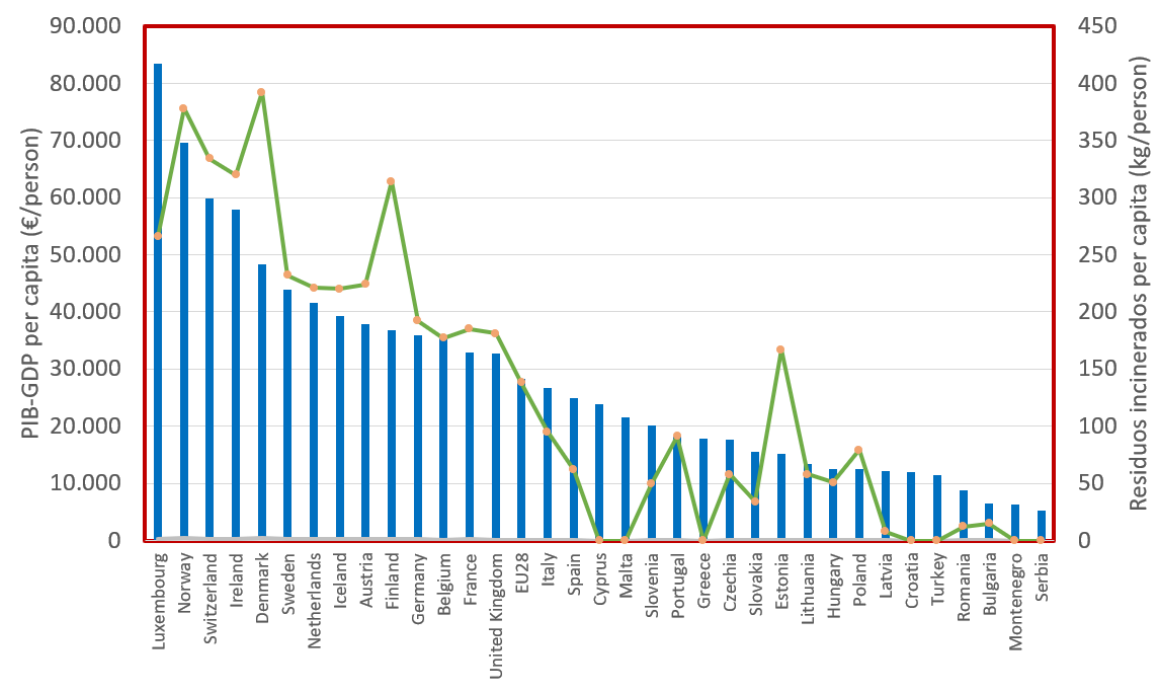

Figure 4.

As a summary of this block of waste treatment, the percentages that could be presented as treatment systems in Spain are 15\% recycling (metal and plastics), 18\% methanation or composting, 9\% incineration and $58 \%$ landfill.

But ... Why European policy is zero landfill? I mean, why is the spill so dangerous? In the first place, the smell and the visual contamination, that would be almost the least important, although the residents of these landfills have to suffer it . Second, there is the issue of leachate, which is the rainwater collected in the deepest (waterproofed) areas. By their nature, these waters have a high content of heavy metals and organic acids, which makes them very dangerous. In the current legislation, the destination of this leachate must be to irrigate again the upper layers of the landfill vessel.

There is also the problem of birds addicted to eating junk [3]. The decrease in the size of birds' eggs and their chicks has been related to the proximity to landfills [4]. The levels of dioxins and furans and other related compounds have been found to be much higher in birds living in landfill areas [5]. We must bear in mind that they eat things that are contaminated.

We must also bear in mind that a highly flammable gas is produced in landfills, mostly consisting of methane. This methane of course if there is a spark is going to catch fire, and will produce a very difficult flame to extinguish. In addition, sometimes (not very rare) self-combustion of the materials that constitute the landfill can occur, since it can occur in certain concentrations of organic matter and the increase in temperature that occurs due to natural fermentation. As we also know, the oxygen supply in these conditions and the temperature control are very poor, if not non-existent. These are the ideal conditions for poor combustion to occur with the production of high levels of organic pollutants, including dioxins and furans [6].

Other news related to landfills try to count the illegal landfills. These area a very big environmental problem. If the legal ones already present big problems, the simple fact of throwing a residue to certain places is a great environmental attack. 
At this point we ask... why incineration is the preferred system for the EU to treat the residual fraction? Note that in the pyramid that we have seen previously, incineration is preferred to landfilling. This is due to the fact that the necessary technology exists for the treatment of the gas produced in the incinerator, and that there is a control of the temperature and conditions in which the combustion occurs. It is true that incineration produces a final solid residue, which must be disposed of, and which will probably be the manufacture of ceramic bricks. The most dangerous element of an incinerator, although it is not known to the general public, is fly ash. It is as very fine ash that have been entrained by the gas, then collected, and exhibiting very high content of dioxins and furans. The most logical thing is to immobilize these ashes as soon as possible, in matrices such as mortars or cements, so that they completely lose their danger. Finally, indicate that the incinerators have a very interesting performance in electrical production.

The case of the Vienna incinerator is well known for being a facility that is in the middle of the city and has been operating for many years. There has been no problem with this facility, where epidemiological studies and population monitoring have been done to check for danger, and where no further incidence of cancers or other diseases has been detected. Of course, these facilities are subject to periodic controls of pollutants, which must be followed for the tranquility of the population.

\section{PLASTIC WASTE}

Plastic waste is especially problematic. There are estimates that around 10 million tons of plastic reach the sea each year. This, if we take into account the world population and making a simple division, corresponds to $1.5 \mathrm{~kg}$ of plastic per person on this planet per year. To get an idea, this means that of the $500 \mathrm{~kg}$ of garbage that we generate per person in Spain per year, $1.5 \mathrm{~kg}$ is plastic that reaches the sea. It's a lot? Is it little? Well, it is a reflection topic. The figures are there.

Why do arrive plastic sea? First, plastics are almost indestructible. Last week my son, 5th grade, told me that his teacher told them that a plastic doesn't decompose until 100 years have passed. Not so, the figure is much higher and the worst thing is that the destruction, as we will see, does not make the plastic disappear, but that increasingly smaller, invisible particles can be produced, which persist for many centuries.

Second reason, they are light, they do not weigh. This makes the air or water currents take them to the natural sink of the whole world that is the sea. We will see below some transport mechanisms.

Another common reason is that a lot of plastic is produced, but is a lot of plastic really produced? And the last question is the one already mentioned about the poor treatment of waste.

Let's look at some figures in Figure 5. A few days ago I started collecting data on products that are likely to be produced in large quantities. We see the data. If there is something in this world that is produced a lot, it is cement. 4,000 million tons a year. Wood in fibers, around 500 ton / year ... The global figure for plastics is approx. 350 ton. We see that more wood is produced ... but of course, this is biodegradable and is "friendly". 


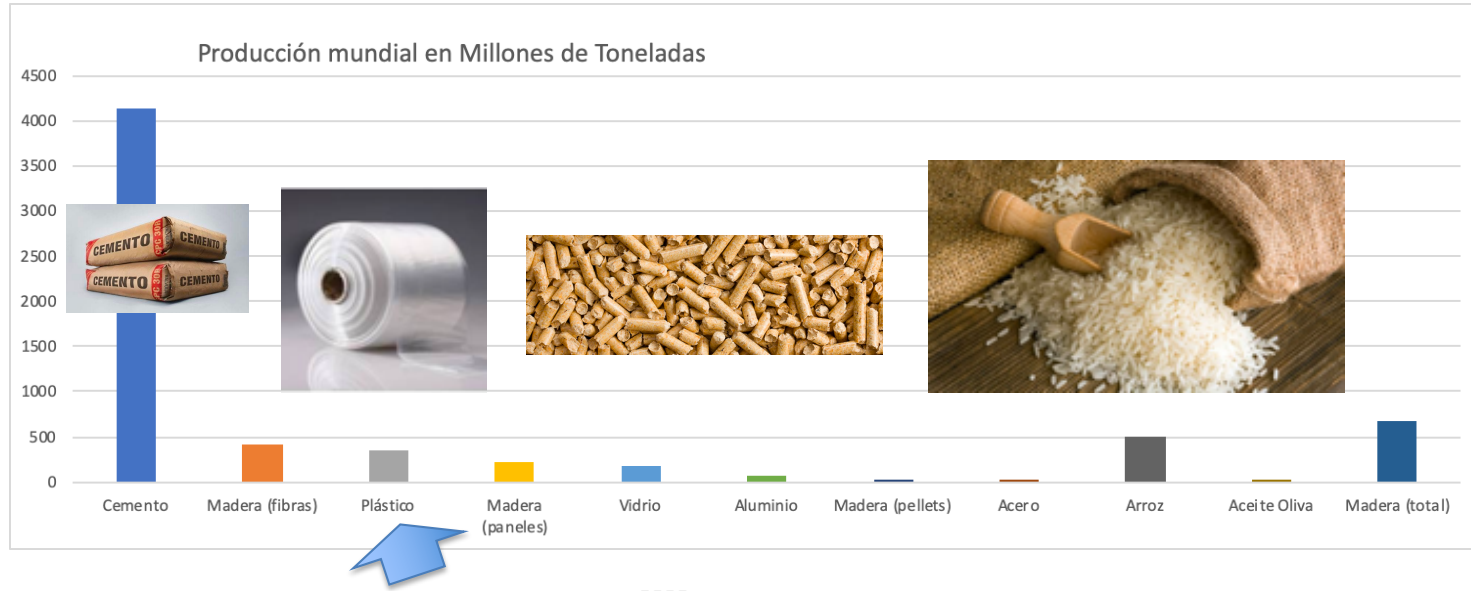

348 million tonnes

Figure 5.

Second question, is the plastic treated properly? Well we have seen that not, but also it is not even deposited properly. We all have seen containers in our cities surrounded by plastics that have not even been deposited inside. It is also true that the problem is not exclusive to plastics.

It is true that the recycling rate of plastics has been increasing, with figures ranging from $24 \%$ in 2005 to $43 \%$ in 2016 [7], but still much remains to be done. In addition, these figures must be taken with great care because depending on the source in question (producers, environmentalists, pressure groups, ...) we are going to find very different figures.

This deficient treatment what it produces is that the residue reaches the sea through various mechanisms as we will comment: by human action, by rivers, winds, "accidental" loss... forming what are called "marine debris" that contain many plastics, but also wood and other components.

The mechanisms by which these plastic wastes reach the sea are well studied. There are many routes of entry, among which I would highlight what is called the "urban runoff". This is simply rainwater carrying plastics and other debris. Let's think about episodes of abundant rains as it is happening, unfortunately, on many occasions. Some organizations, town halls and irrigation communities are trying to implement some devices that prevent the solids being washed from reaching the sea. They are introduced into spillways where the water goes to the sea.

\section{MICROPLASTICS}

The plastic problem is no longer that of the "macroplastic" but that this waste is going to go debasing, is becoming smaller, until it forms what is called "microplastic" which are very small particles. We have already said that they are practically indestructible ...

But... what are microplastics? There is no final consensus on the definition. It is clear that they are very small plastics, but the limit is not too agreed. In general, it is admitted that they are plastic particles whose size is between $5 \mathrm{~mm}$ and 100 microns. If they are older, it would be macroplastics and if it is below, it is called nanoplastics. Let's see that a speck of dust has approx. 500 microns. In other words, we are talking about practically invisible particles. 
Some headlines in the press produce confusion more than anything. It is easy to find headlines of the type "Collection of microplastics on the beach" or similar. Journalism insists that microplastics can be collected without much effort, but given the definition we have just discussed, we see that it is very likely that what is collected on the beach is not microplastics, but this contributes to the general chaos in this type of themes. Of course, in this case, the initiative is good but microplastics are not collected.

Apart from the polymer that constitutes them, if it is PE, PVC ... microplastics are classified as primary and secondary. The primary would be those that are already manufactured as microplastic. Now there are more restrictions for its manufacture, but they have generally been used in hygiene products (exfoliating creams, toothpaste, gels, ...). The secondary microplastics are those that we have discussed derive from the wear or degradation of large plastics (heat, UV, mechanical or biological degradation).

They are also classified by the shape they have. There would be fragments (larger, we would almost say they are macroplastics), microfibers (with an elongated shape, seen under a microscope), microspheres and microbeads that are small spheres, there are also foams and pellets. Between pellets, microspheres and microbeads the differences are very subtle. We would say then that there are microfibers, elongated, and microparticles of spherical or undefined shape. We have an example in Figure 6.
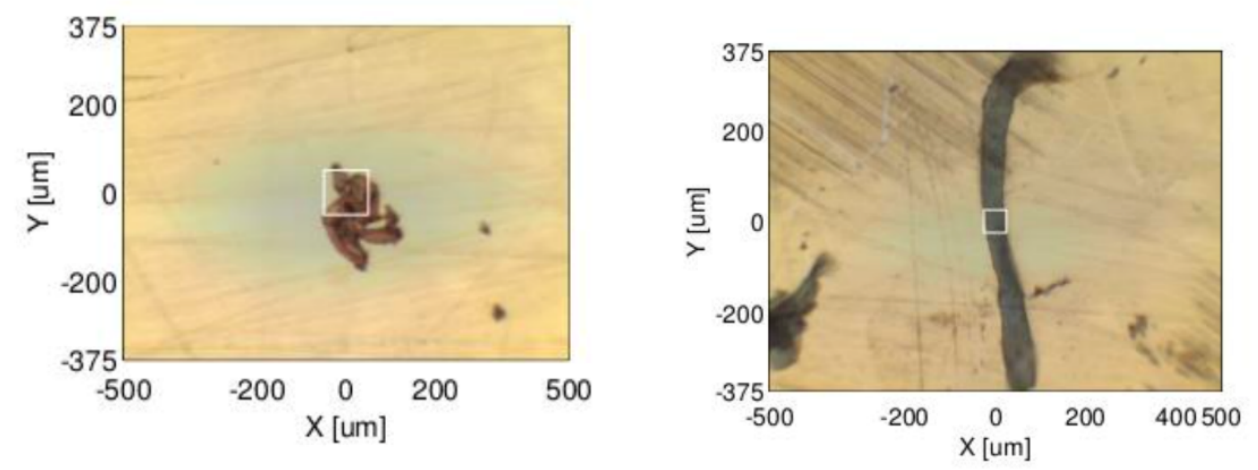

Figure 6.

We have already discussed what the sources are, but by commenting on something else, the accidental discharge of primary microplastics would be possible, and another possible source that is increasingly being talked about is garment washing water. If the clothes are made of polyester, this is exactly the same as PET and it is already in the form of fibers, which proves that they can be a big problem. There are already manufacturers of washing machines that indicate that they have filters to avoid the discharge of microplastics.

Let's see what happens to microplastics in wastewater treatment systems. It has been seen in a study [8] (South Korea) that in a conventional system $75 \%$ of microplastics are removed in primary treatment. In addition, it is increased to $95 \%$ in secondary treatment. Advanced tertiary treatment would be needed to increase it to $98 \%$. Within this treatment, sand filtration (which is expensive), 
electrocoagulation (much more expensive) stand out. Thus, in general, there is no specific microplastic treatment system in plants, but there is a certain reduction in them. Although these figures will surely be very little reproducible in other systems. Thus, the purified water will contain a quantity (important or not) of microplastics, which will be used to irrigate crops, or will be taken directly to the sea.

Later we will talk about the presence of microplastics in food, but let's see what effects their presence in the sea can have. The first would be the blockage of the intestinal tract of the fish, but this would be more restricted to macroplastics. The presence of nanoplastics in water has been shown to decrease the effectiveness of algae photosynthesis, but in systems where these particles are added in significant amounts. What is true is that, apart from their existence in themselves, plastic microparticles, due to their surface tension and surface properties, attract other molecules that are most likely formed by pollutants. Thus, it has been seen that the concentration of PAHs and PCBs in microplastics in water is 1 million times greater than that of seawater; As we will see, fish can eliminate part of the plastics they eat, but not those contaminants.

In fact, an increase in the levels of microplastics in the food chain has been observed, that is, they are not easily eliminated either. That is, that the larger fish have higher levels of microplastics than the smaller ones,...

Regarding the effect of microplastics in humans, there is still no evidence of biological effects. There are no conclusions. It is true that if plastics have other contaminants attached, it would be an added problem. Plastic as such will likely pass through the digestive tract and be excreted, in fact, they have been found in human feces.

How are microplastics analyzed? A procedure is carried out that includes the preparation of the sample, the isolation of the microplastics, their counting and the determination of their nature (identification).

As for the sample preparation, it depends a lot on the form of the food. For water and soluble substances, there is simply a filter, of adequate pore size, in which the particles will be retained. Other liquids such as oils, beer, wines, juices, ... would also be analyzed as well. Other similar methods would be flotation and sedimentation. When the matrix is not soluble, either the animal is dissected looking for the plastics and separate them, or they are digested with basic, acidic or enzymatic treatments. These treatments can eliminate some plastics, depending on their nature, and it must be taken into account.

One of the problems that arise is the existence of natural fibers that with most of the methods are going to be isolated as well, false positives would occur. For example, in honeys and sugars it is very common, and a treatment with hydrogen peroxide would be carried out to discolor the natural fibers and know it at the time of identification. There are also selective dyes such as Nile Red or Bengal Pink that allow us to distinguish between natural and synthetic.

The next thing is, once we have the fiber or plastic insulated, proceed to identify it with some technique. In the UA we have used micro FTIR that allows to make a spectrum of the fiber, one by one (if the signals would not be mixed) and compare with plastic standards. Another similar

*email: ja.conesa at ua.es 
technique is the micro RAMAN, also widely used, which has drawbacks due to the possible fluorescence signal of other elements that do not allow proper identification.

Other identification techniques would be thermal analysis, combining pyrolysis with gas chromatography, but the sample in this case is destroyed and no information is obtained on the number of microplastics, only on their nature. There are many other quite complex techniques to try to analyze particle mixture.

In the UA we have made an analysis of the microplastics contained in many salt samples [9], chosen so that we had samples of sea salt and well salt, with different particle sizes and before and after packaging. The purpose was to obtain information on the origin of these microplastics. For this, as I have mentioned, a certain amount of salt was dissolved in ultrapure water, filtered with a size of 5 microns and brought to the infrared.

The results show that most plastics are microfibers, in fact, we have not seen microspheres. In addition, the predominant colors were red, black, transparent ... and regarding their nature, practically $80 \%$ of those analyzed were PET, followed by PE and PP. This is normal, since they are the most widely used plastics globally, in addition to the fact that PET can come, as we have said, from tissue fibers.

In this study we found was no significant difference between the amount of microplastics contained in sea salt and well salt (Figure 7). This can draw a lot of attention as we would expect more from the salt of sea water. The numbers indicate no, having found levels between 50 and 280 in sea salt and between 115 and 185 Microplastics / $\mathrm{kg}$. We also found that packaging has no effect on the level of plastics, and neither does grinding.

SEA SALT WELL SALT
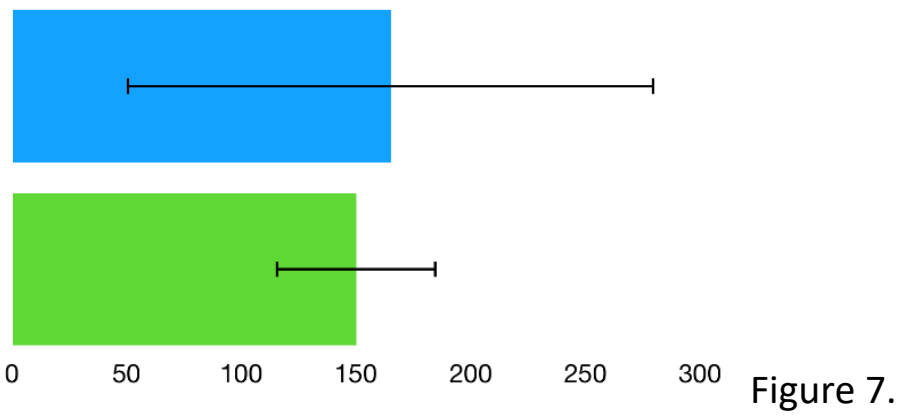

We have analyzed other foods (juices, beers, milk, sugar) ... (see Table) where we find that the quantity is not alarming in any case. In Dead Sea salts we also find a large level, similar to that found in all Spanish salts. They reached our laboratory salts "pharma degree", which are used for serum in hospitals, checking that the levels are much lower than the others, even only 4 particles per $\mathrm{kg}$. These salts are produced in a thermal process, under vacuum in which it is practically treated as a chemical reagent with a highly elaborated system. 
Table 1.

\begin{tabular}{|l|c|}
\hline Sample & $\begin{array}{c}\text { Unit } \\
\text { (items/L) or (items/kg) }\end{array}$ \\
\hline Apple juice & 21 \\
\hline White wine & 36 \\
\hline Red wine & 15 \\
\hline Beer & 45 \\
\hline Sugar & 230 \\
\hline Whole milk & 21 \\
\hline Semi-skimmed milk & 15 \\
\hline Skimmed milk & 4 \\
\hline Soda & 4 \\
\hline Vinegar & 15 \\
\hline Sunflower oil & 6 \\
\hline Red wine with soda & 5 \\
\hline Tonic water & 2 \\
\hline Salt vacuum (pharma grade) & 4 \\
\hline Salt vacuum (in pellets) & $35-45$ \\
\hline Salt from dead see & 225 \\
\hline
\end{tabular}

Comparing our analyzes with salts from other countries, which have been published in scientific literature $[10,11]$, we see that a lot of variety. We have studies of salts from China, with levels around 600-800 particles per $\mathrm{kg}$, and salts of the area of Croatia, where they found thousands of microplastics per kg, even 20000.

All this indicates is that there are: highly contaminated sites, such as salt from Croatia or China, with levels above 600 particles/kg; intermediate systems with levels around 200 items kg; and very little contaminated systems that would be correlated with chemical salts.

We had also the opportunity to analyze different types of salt from a well-known producer who wanted to estimate the weight of these particles in the salt. It is not possible to isolate enough quantity to be able to weigh them, so the most logical thing is to estimate their weight through its dimensions and density. To do this, we made more precise measurements and assumed the worst case, PET, which has the highest density. With this, we conclude that the weight of the fibers is a maximum of 100 micrograms per gram of salt.

We were also able to collect water from the different stages in which the salt is concentrating in the brine, and we proceeded to its analysis. It was able to verify that there is an increase in the amount of microplastics as water has higher content of salt. This may be due to greater exposure to the environment, since if these microparticles come from the air the deposition time will be longer, but it may also be that the initial amount is concentrated so that the concentration is higher per liter of water.

There is a study from 2019 [12], which estimates how many microplastics we can be consuming over the course of a year. With the published data regarding the amount of microplastics containing salt, water, sugar, wine, beer, fish and others, and taking into account the amount ingested of each food annually, the calculation is relevant . In this study, a distinction is made between the sexes, and the 
conclusion is reached that if you only drink tap water, the maximum levels are much lower (around 20,000 particles per year) than if you drink bottled water (reaching 120,000 particles). /year). Logically, bottled water producers did not like it, just as our study at the UA did not like Spanish salt producers, since, in some way, they are put in the crosshairs. With these figures, we can make an estimate of the weight of plastic we eat per year.

Thus, we can see that the global average intake mentioned in the study is 74,000 particles per year. With the aforementioned method, it can be estimated that an average fiber weighs 0.3 micrograms, which would give a total of 20-40 milligrams a year.

In a campaign promoted by WWF it is said that each human eats a credit card ( $5 \mathrm{~g})$ a week with the food we eat. This has been reproduced, and continues to be, in a multitude of media. This headline is absurd to me, but shocking, that is, journalistically perfect! The journalist wants us to read it, and if we see a headline that says this, it is impossible not to see the complete report. If 74000 particles/year supposes 5 grams per week, then a microparticle weighs 3.54 milligrams. This is absurd. Each particle weighs micrograms, not milligrams ... an error of 1000 orders of magnitude. This shows that just finding something on the internet does not mean it is true ... among other things.

Other headlines related to the topic: "In 2050 there will be more plastics than fish in the sea", this time we can agree, but we consider that a single plastic particle, for example $1 \mathrm{~g}$, can break into several, and these in others, and others, and others, so that only $1 \mathrm{~g}$ can count as many particles. In the press headline it is not said that the plastic will be weighed more, it only talks about the number of plastic and fish. It is also true that this gives us another lesson: a single particle of plastic that is not treated well can contaminate millions of microplastics that nature will return to us with salt, or fish....

In the UA we continue to work with the microplastics on two fronts. On the one hand, we are trying to extract from these microplastics and determine contaminants associated with microplastics, since we have the appropriate techniques. And on the other, we are investigating the effectiveness of different sand beds with different sizes and types of plastics.

In closing, since the conclusions I already said at the beginning, insist with some messages to take home. The first is that Spain (Europe in general) is very deficient in waste treatment. Second, incineration is not the worst treatment system. Third, there are microplastics even in the soup, literally. It is also true that the quantity is not important, at the moment, and that the effect it can have on humans is not known. We have also seen that contamination by microplastics food can be great in highly contaminated areas across the little contaminated, but microplastics absolute zero is unattainable.

\section{References}

1. EUROSTAT Municipal waste statistics.

2. European Parliament and of the Council Directive 2008/98 / EC. Off. J. Eur. Union 2008 , L 312 , 330. 
3. Gilbert, NI; Correia, RA; Silva, JP; Pacheco, C.; Catry, I .; Atkinson, PW; Gill, JA; Aldina, AM Are white storks addicted to junk food? Impacts of landfill use on the movement and behavior of resident white storks (Ciconia ciconia) from a partially migratory population. Mov. Ecol. 2015 , 4 , 1-13, doi: 10.1186 / s40462-016-0070-0.

4. Tongue, ADW; Reynolds, SJ; Fernie, KJ; Harrad, S. Flame retardant concentrations and profiles in wild birds associated with landfill: A critical review. Environ. Pollut. 2019 , 248, 646-658, doi: 10.1016 / j.envpol.2019.01.103.

5. Guigueno, MF; Fernie, KJ Birds and flame retardants: A review of the toxic effects on birds of historical and novel flame retardants. Environ. Res. 2017 , 154 , 398-424, doi: 10.1016 / j.envres.2016.12.033.

6. Stanmore, BR The formation of dioxins in combustion systems. Combust. Flame 2004 , 136 , 398427.

7. Packaging waste statistics - Statistics Explained Available online: https://ec.europa.eu/eurostat/statistics-explained/index.php/Packaging_waste_statistics (accessed on Mar 12, 2020).

8. Lv, X .; Dong, Q .; Zuo, Z .; Liu, Y .; Huang, X .; Wu, WM Microplastics in a municipal wastewater treatment plant: Fate, dynamic distribution, removal efficiencies, and control strategies. J. Clean. Prod. 2019 , 225 , 579-586, doi: 10.1016 / j.jclepro.2019.03.321.

9. Iñiguez, ME; Conesa, JA; Fullana, A. Microplastics in Spanish Table Salt. Sci. Rep. 2017 , 7 , 1-7, doi: 10.1038 / s41598-017-09128-x.

10. Yang, D .; Shi, H .; Li, L .; Li, J .; Jabeen, K .; Kolandhasamy, P. Microplastic Pollution in Table Salts from China. Environ. Sci. Technol. 2015 , doi: 10.1021 / acs.est.5b03163.

11. Renzi, M .; Blašković, A. Litter \& amp; microplastics features in table salts from marine origin: Italian versus Croatian brands. Mar. Pollut. Bull. 2018 , 135 , 62-68, doi: 10.1016 / j.marpolbul.2018.06.065.

12. Cox, KD; Covernton, GA; Davies, HL; Dower, JF; Juanes, F .; Doubts, SE Human Consumption of Microplastics. Environ. Sci. Technol. 2019 , acs.est.9b01517, doi: 10.1021 / acs.est.9b01517. 